\title{
Photon Emission Spectroscopy of Single Oxide-Supported Ag-Au Alloy Clusters
}

\author{
W. Benten, N. Nilius,* N. Ernst, and H.-J. Freund \\ Fritz-Haber-Institut der Max-Planck-Gesellschaft, Faradayweg 4-6, D-14195 Berlin, Germany
}

(Received 22 July 2004; revised manuscript received 29 November 2004; published 1 July 2005)

\begin{abstract}
The alloying of $\mathrm{Ag}$ and $\mathrm{Au}$ has been investigated on the level of individual clusters by analyzing the light emission excited by electron injection from an STM tip. Different Ag-Au alloy and shell-core clusters have been prepared at room temperature on a thin $\mathrm{Al}_{2} \mathrm{O}_{3}$ film on $\mathrm{NiAl}(110)$ by simultaneous and successive deposition of both noble metals. For simultaneous deposition, one Mie-plasmon resonance has been detected with a wavelength position shifting from the pure Au to the Ag value with increasing Ag content. The results are in agreement with calculations based on Mie theory indicating a complete mixing of both materials. For successive deposition, two Mie resonances have been observed, attributed to plasmon excitations in the shell and core of the clusters. Comparing these results to model calculations, a considerable intermixing of the core and shell materials is concluded, which is especially strong in Au shell-Ag core clusters.
\end{abstract}

DOI: 10.1103/PhysRevB.72.045403

PACS number(s): 68.47.Jn, 61.66.Dk, 68.37.Ef, 78.67.Bf

\section{INTRODUCTION}

Industrial catalysts composed of oxide supported alloy particles often show a strongly enhanced chemical reactivity compared to systems containing only monoelemental aggregates. ${ }^{1,2}$ The outstanding properties of alloys in heterogeneous catalysis result from a number of effects, e.g., a lowered barrier for a specific chemical reaction, an increased selectivity, ${ }^{3,4}$ and an enhanced resistance against contamination and poisoning. ${ }^{5,6}$ The formation of alloy particles on thin oxide films has recently been characterized using scanning tunneling microscopy (STM) and electron and infrared spectroscopy. ${ }^{7-9}$ In contrast to bulk alloys, the intermixing in small particles depends on several additional parameters, such as diffusivity, nucleation and growth of the compounds on the oxide support, their surface free energy and miscibility, and the thermodynamic alloying conditions. As a result, it often remains unclear if the compounds completely mix to alloy clusters, form shell-core structures with different composition of inner and outer layer or separate into monoelemental clusters on the surface.

The intermixing of different metals to alloy particles can in principle be followed by changes in their optical characteristics. Optical properties, especially of noble metal clusters, are dominated by strong extinction peaks, caused by the excitation of collective electronic modes, so-called Mie plasmons. ${ }^{10}$ Resonance energy and linewidth of these excitations distinctively depend on size, shape and environment of the clusters, but also on their exact chemical composition. Mie-plasmon excitations in pure Ag and Au particles are well separated in energy, but converge with an increasing alloying of both materials. ${ }^{11-13}$ The analysis of Mie plasmons in mixed Ag-Au particles is therefore a sensitive means to characterize the alloying process.

Mixed Ag-Au particles have intensively been studied by various experimental and theoretical techniques. Due to their perfect miscibility and distinct optical properties, they represent a model system to obtain a principle understanding of the alloy formation in small particles. Mixed $\mathrm{Ag}$-Au colloids have been prepared in aqueous solution,,${ }^{11,14-18}$ and solid matrices, such as glass, ${ }^{12,13}$ silica, ${ }^{19}$ and alumina. ${ }^{20}$ Optical ab- sorption and extinction spectroscopy was employed to analyze the cluster ensembles. Since these classical optical techniques average over millions of particles with different sizes, shapes, and compositions, the resulting spectra are affected by a considerable inhomogeneous broadening. The competition between alloying, shell-core formation and phase separation in the particles cannot be deduced as a function of cluster size and details of the alloying process might be hidden in the ensemble spectra.

To circumvent these problems we have combined photon emission spectroscopy and scanning tunneling microscopy to investigate the alloying of $\mathrm{Ag}$ and $\mathrm{Au}$ in individual clusters on a $\mathrm{Al}_{2} \mathrm{O}_{3} / \mathrm{NiAl}(110)$ surface. In the experiments, the STM tip injects electrons into selected clusters to excite Mie plasmons. Photons emitted due to the radiative plasmon decay are analyzed according to their wavelength. ${ }^{21}$ From peak position and linewidth of the plasmon modes, chemical composition and alloying state of the clusters are determined. The results are compared to measurements on $\mathrm{Ag}$ - $\mathrm{Au}$ particle ensembles and calculations based on Mie theory.

\section{EXPERIMENTAL}

The experiments were carried out in an UHV chamber $\left(p<2 \times 10^{-10} \mathrm{mbar}\right)$, equipped with a Beetle-type STM and standard surface-science methods for sample preparation and analysis. The STM tip was located in the focal point of a parabolic mirror, which collected photons emitted from the tunneling junction and reflected them out of the chamber. After passing a quartz window, the light was focused by a second mirror onto the entrance slit of a grating spectrograph and detected with a liquid-nitrogen cooled CCD detector. Details of the experimental setup have been described earlier. $^{22}$

Mixed Ag and Au clusters were prepared by atom deposition from the gas phase onto a thin, well-ordered alumina film at $300 \mathrm{~K}$. The film was grown by exposing a clean $\mathrm{NiAl}(110)$ surface to $1200 \mathrm{~L}$ of $\mathrm{O}_{2}$ at $550 \mathrm{~K}$. Subsequent annealing of the sample to $1100 \mathrm{~K}$ lead to the formation of a well-ordered alumina film with a thickness of approximately 
$0.5 \mathrm{~nm}$, which is suitable for STM characterization. ${ }^{23,24} \mathrm{Be}-$ cause of a relatively weak metal-oxide interaction, $\mathrm{Ag}$ and Au grow to 3D clusters at $300 \mathrm{~K}$ (Volmer-Weber growth). Alloy clusters were prepared by simultaneous deposition of the two metals, shell-core clusters by successive deposition of both materials interrupted by a 15-min break. The total amount of deposited material in alloy and shell-core clusters was 3.5 and 4 monolayer (ML), respectively. ${ }^{25}$

Photon emission spectra were measured for single $\mathrm{Ag}-\mathrm{Au}$ clusters selected from topographic STM images. Morphological changes of the examined clusters or drift of the tip position during the measurement could be excluded through repeated imaging of the same surface region after each spectroscopic run. The photon emission spectra were taken with an electron current of $4 \mathrm{nA}$ and a tip voltage larger than $-13 \mathrm{~V}$. The tip-sample distance at these conditions was estimated to be $2 \mathrm{~nm}$, minimizing the influence of the tip on the cluster electronic system. The diameter of the electron beam was smaller than the mean cluster-cluster distance and excitation of single clusters could be ensured. A negative tip voltage was chosen to inject electrons into the clusters. The accumulation time for each photon emission spectrum ranged between 300 and $600 \mathrm{~s}$. An entrance slit width of 1 $\mathrm{mm}$ was used for the spectrograph.

\section{RESULTS AND DISCUSSION}

\section{A. Nucleation and growth of Ag-Au clusters}

STM images in Figs. 1(a)-1(d) show Ag-Au alloy clusters on $\mathrm{Al}_{2} \mathrm{O}_{3} / \mathrm{NiAl}(110)$ with an increasing $\mathrm{Ag}$ content at constant total coverage of $3.5 \mathrm{ML}$. With increasing Ag content, the density of clusters decreases while the mean cluster size increases. Ag-rich particles have almost hemispherical shapes with an average height of 5-6 nm. The aspect ratio (height/diameter) was determined to be approximately 0.5 . An increasing $\mathrm{Au}$ fraction leads to the growth of clusters with distinct polygonal geometry. For Au-rich clusters, the height successively decreases to $2 \mathrm{~nm}$ with a mean diameter of $8 \mathrm{~nm} .{ }^{26}$ This change in cluster shape is reflected in a smaller aspect ratio of 0.3 for the Au-rich particles. Figures 1(e) and 1(f) show STM images of Ag shell-Au core clusters with different compositions at a total coverage of 4 ML. The shell-core clusters with a higher amount of Au grow with higher density and pronounced polygonal shapes, while Agrich particles have more hemispherical shapes. In the opposite case of Au shell-Ag core clusters, the clusters are generally more hemispherical in shape (not shown).

The STM images show that cluster shapes sensitively depend on the Ag-Au mixing ratio. The presence of welldefined cluster shapes indicates a crystalline structure. We suggest a [111] orientation for the polygonal surfaces observed on pure $\mathrm{Au}$ and $\mathrm{Ag}$-Au alloy clusters. Fcc-metals such as $\mathrm{Ag}, \mathrm{Au}, \mathrm{Pd}$, and Pt preferentially grow with [111] orientation due to the low surface free energy of their (111) surfaces. For Pd clusters on $\mathrm{Al}_{2} \mathrm{O}_{3} / \mathrm{NiAl}$, the [111] orientation of the top facet could be verified by atomically resolved STM measurements. ${ }^{27}$ Molecular dynamic simulations of Ag and $\mathrm{Au}$ particles also found a preferential development of (111) surfaces at room temperature. ${ }^{28}$
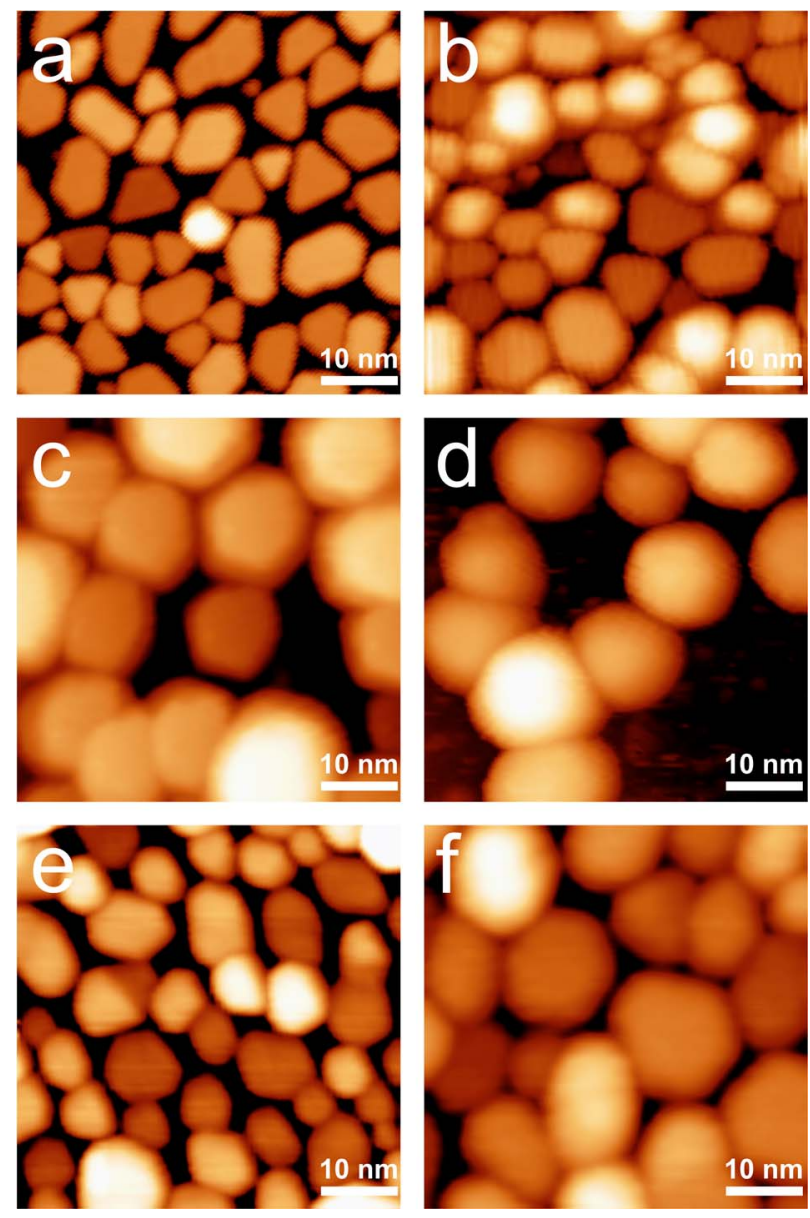

FIG. 1. (Color online) STM images of (a)-(d) Ag-Au alloy clusters at 3.5 ML total coverage and (e), (f) shell-core clusters at $4 \mathrm{ML}$ total coverage on $\mathrm{Al}_{2} \mathrm{O}_{3} / \mathrm{NiAl}(110)$ (Image sizes: $50 \mathrm{~nm} \times 50 \mathrm{~nm}$ ). The Ag content is $0 \%$ in (a), $40 \pm 5 \%$ in (b), $70 \pm 4 \%$ in (c), and $100 \%$ in (d). Ag shell-Au core clusters with $25 \pm 4 \%$ and $50 \pm 5 \% \mathrm{Ag}$ fraction are shown in (e) and (f), respectively. All images have been obtained at $-1.5 \mathrm{~V}$ tip bias and $0.1 \mathrm{nA}$ tunneling current at $300 \mathrm{~K}$.

In our experiments, pure $\mathrm{Ag}$ clusters preferentially grow at step edges and domain boundaries of the alumina film, while pure Au clusters also nucleate on oxide terraces. The increase in cluster density for $\mathrm{Ag}-\mathrm{Au}$ alloy clusters with increasing $\mathrm{Au}$ fraction indicates a lower mobility of $\mathrm{Au}$ atoms on the thin $\mathrm{Al}_{2} \mathrm{O}_{3}$ film compared to $\mathrm{Ag}$ atoms. Also the smaller aspect ratio of $\mathrm{Au}$ compared to $\mathrm{Ag}$ particles points to an increased Au-oxide interaction strength. Similar observations were made on $\mathrm{TiO}_{2}(110)$ surfaces, where Ag clusters grow with larger average sizes and lower densities than $\mathrm{Au}$ clusters. ${ }^{9}$ STM images of shell-core clusters suggest that the metal deposited first, i.e., the cluster core, determines shape, size, and density of the clusters. The Ag shell-Au core clusters are consequently flatter and grow with a higher density compared to the reverse configuration.

\section{B. Optical properties of Ag-Au alloy clusters}

Photon emission spectra of different Ag-Au alloy clusters with a $\mathrm{Ag}$ content of $70 \pm 4 \%$ are shown in Fig. 2. The spec- 


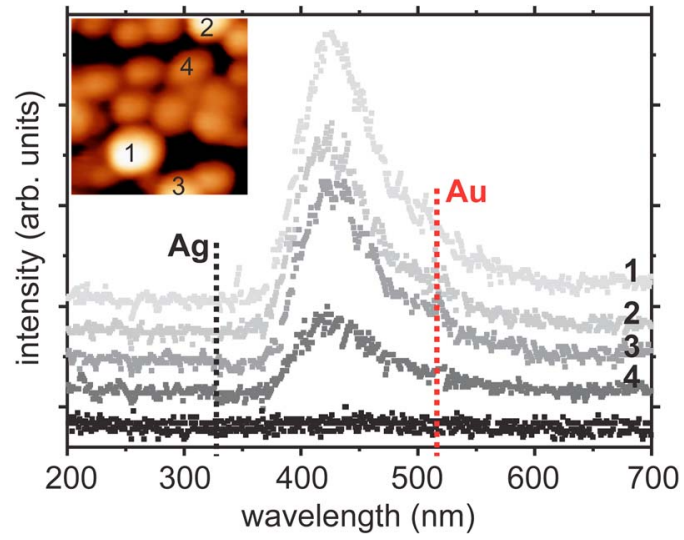

FIG. 2. (Color online) Photon emission spectra of differently sized $\mathrm{Ag}$-Au alloy clusters on $\mathrm{Al}_{2} \mathrm{O}_{3} / \mathrm{NiAl}(110)$ with $70 \pm 4 \% \mathrm{Ag}$ content. Corrected particle sizes range between 6-10 nm. Spectra were measured on top of the labeled clusters in the STM image shown in the inset $(50 \mathrm{~nm} \times 50 \mathrm{~nm})$. A spectrum for the bare alumina film is shown at the bottom $\left(U_{\text {tip }}=-14 \mathrm{~V}, I=4 \mathrm{nA}\right)$.

tra were measured on top of the clusters indicated in the STM image in the inset. They exhibit an intense emission line centered at around $425 \mathrm{~nm}$. The peak position is located in between the values obtained for pure $\mathrm{Ag}$ and $\mathrm{Au}$ clusters of approximately 330 and $510 \mathrm{~nm}$, respectively. Clusters of bigger size show higher peak intensities, however, no dependence of the peak position on the cluster size could be found. At the bottom of the figure, a photon emission spectrum of the bare alumina film is shown, where no emission is observed in the analyzed wavelength region. For an increasing $\mathrm{Ag}$ fraction in the Ag-Au alloy clusters, the position of the emission peak monotonously blueshifts from the value of pure $\mathrm{Au}$ to the one of pure Ag clusters (Fig. 3). Simultaneously, the linewidth decreases with increasing Ag fraction from about 115 to $30 \mathrm{~nm}$ FWHM.

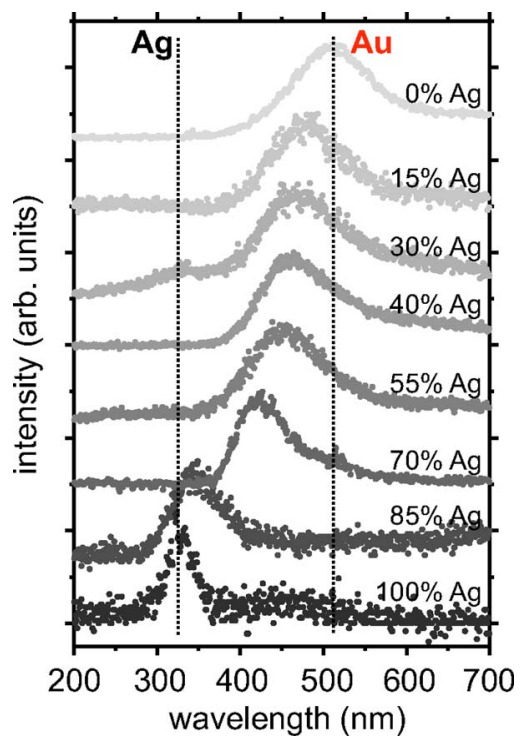

FIG. 3. (Color online) Normalized photon emission spectra of single $\mathrm{Ag}-\mathrm{Au}$ alloy clusters on $\mathrm{Al}_{2} \mathrm{O}_{3} / \mathrm{NiAl}(110)$. The $\mathrm{Ag}$ content increases from $0 \%$ (top) to $100 \%$ (bottom) $\left(U_{\text {tip }}<-13 \mathrm{~V}, I=4 \mathrm{nA}\right)$.
The optical spectra of Ag-Au alloy clusters reveal pronounced emission intensity for tip voltages larger than $-13 \mathrm{~V}$, when the tip-cluster distance is relatively large. This behavior is not compatible with an emission process mediated by tip-induced plasmons (TIP), where a localized electromagnetic mode is formed in the cavity between tip and sample. At the relatively high voltages used in the experiments, the excitation cross section for TIP's is rather small and light emission from this channel becomes weak. ${ }^{29} \mathrm{We}$ therefore propose an emission mechanism, which is dominated by the radiative decay of Mie plasmons in the individual Ag-Au clusters. The plasmon excitation can be interpreted as a collective oscillation of the electron gas versus the ion cores in the clusters and is driven by electron injection from the tip. For supported metal clusters, the Mie resonance splits into two modes, oriented parallel $(1,1)$ and perpendicular $(1,0)$ to the substrate. ${ }^{10}$ Due to the experimental arrangement, only the $(1,0)$ mode is detected, because the electron injection from the tip follows the surface normal and excites primarily this plasmon mode.

The energy position of the plasmon is strongly influenced by the dielectric properties of the alloy clusters and consequently shifts with preceding Ag-Au alloying process. An estimation of the plasmon energy as a function of cluster composition is obtained from calculating the polarizability of a metal spheroid embedded in a dielectric medium ${ }^{10,35}$

$$
\alpha_{(1,0)}(\omega) \propto \frac{\epsilon(\omega)-\epsilon_{m}(\omega)}{\epsilon_{m}(\omega)+L_{\perp}\left[\epsilon(\omega)-\epsilon_{m}(\omega)\right]} .
$$

The eccentricity of the spheroid perpendicular to the surface [i.e., for the $(1,0)$ mode] is expressed by the geometrical factor $L_{\perp}$, calculated from the measured aspect ratios. ${ }^{35}$ The material properties of the alloy are introduced via the experimentally determined dielectric function $\epsilon(\omega)$ for thin $\mathrm{Ag}-\mathrm{Au}$ foils. ${ }^{30}$ The value $\epsilon_{m}$ reflects the dielectric properties of the environment and is modeled in two different ways. In the first case, the environment is described as a linear combination of $70 \%$ vacuum $\left(\epsilon_{\mathrm{vac}}=1\right)$ and $30 \%$ alumina $\left(\epsilon_{\mathrm{Al}_{2} \mathrm{O}_{3}}\right.$ $=3.13) .{ }^{31}$ In the second case, the NiAl substrate underneath the oxide layer is included, resulting in an $\epsilon_{m}(\omega)$ of $0.7 \epsilon_{\mathrm{vac}}+0.15 \epsilon_{\mathrm{Al}_{2} \mathrm{O}_{3}}+0.15 \epsilon_{\mathrm{NiAl}}(\omega) .{ }^{32,33}$ The model can only provide a crude approximation of the experimental situation. The cluster surrounding is treated as a homogenous medium and does not accurately reflect the structure of the metal/ oxide support. In addition, the calculated polarizability gives a measure of the absorption characteristics of the spheroids, including contributions from collective electronic modes and single-electron excitations, such as interband transitions. The measured emission spectra contain exclusively information on collective electronic modes due to their extremely high oscillator strength. In spite of these limitations, the calculations reflect general trends in the Mie-plasmon energy evolving as a function of the Ag-Au mixing ratio and provide a qualitative interpretation of the experimental data. In correspondence to the emission spectra, the calculated $(1,0)$ polarizability of an alloy spheroid shows a single maximum, which indicates the position of the Mie plasmon. For both dielectric environments tested, the plasmon position continu- 


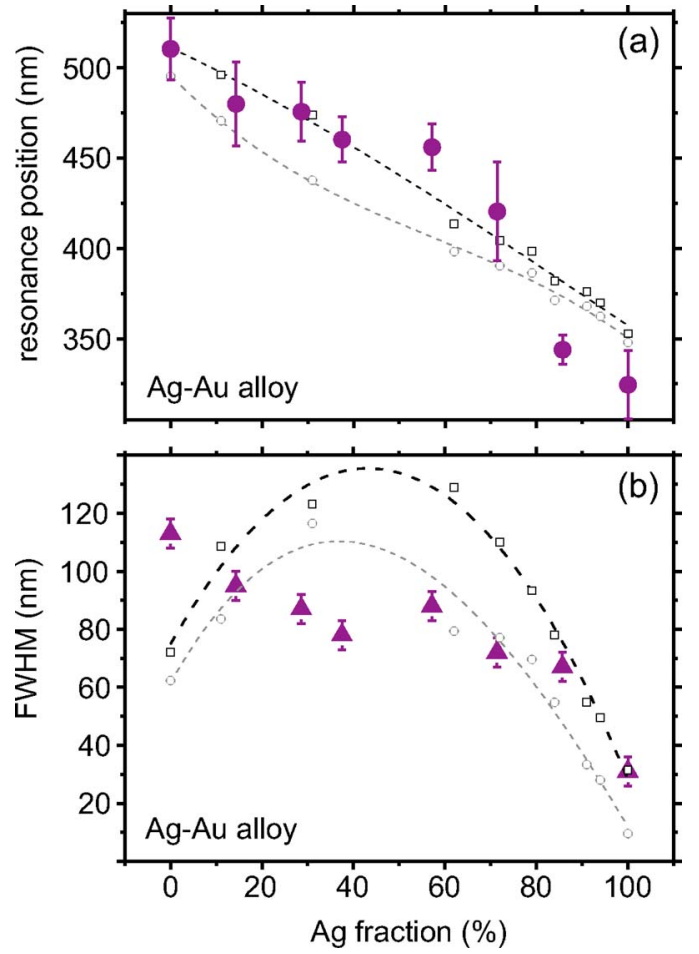

FIG. 4. (Color online) Resonance peak position (a) and FWHM (b) for Ag-Au alloy clusters as a function of $\mathrm{Ag}$ fraction. Closed symbols display the experimental data, open symbols depict values calculated from Mie theory using an experimentally determined alloy dielectric function. The environment of the clusters is assumed to consist of $70 \%$ vaccum and $30 \% \mathrm{Al}_{2} \mathrm{O}_{3}$ (open circles) or $70 \%$ vacuum, $15 \% \mathrm{Al}_{2} \mathrm{O}_{3}$ and $15 \% \mathrm{NiAl}$ (open squares). The dashed lines provide a guide to the eye for the calculated data.

ously shifts to lower wavelengths with increasing Ag amount in the clusters [Fig. 4(a)]. In the first model, which neglects the presence of the NiAl support, the peak positions are generally located at smaller wavelengths than in the experiment. Turning on the NiAl dielectric properties in the second model leads to a red shift of the plasmon resonance and a better reproduction of the experimental peak positions. This shift is not so much induced by differences in the real part of the dielectric functions of $\mathrm{NiAl}$ and $\mathrm{Al}_{2} \mathrm{O}_{3}$. The dominant contribution is owed to the strong absorption coefficient of the metal substrate, which systematically shifts the plasmon to larger wavelengths. Modeling the alloy particles as spheres causes an additional redshift of the $(1,0)$ Mie mode, thus increasing the discrepancy to the measurements. The comparison between calculated and experimental data has to remain on a qualitative level and can only reflect the general trend in the optical shifts with preceding alloying of gold and silver. A more sophisticated model has to include additional effects, such as more realistic cluster shapes, an inhomogeneous cluster environment and the influence of interactions between neighboring particles on the surface. ${ }^{10}$

A strong dependence of the plasmon energy on the composition has earlier been observed for ensembles of $\mathrm{Ag}-\mathrm{Au}$ alloy clusters embedded in photosensitive glass. ${ }^{12,13}$ The extinction spectra were also characterized by a single resonance, which monotonously shifted from the $\mathrm{Au}(510 \mathrm{~nm})$ to the Ag $(405 \mathrm{~nm})$ position with increasing Ag fraction. Our results on single clusters are in line with this observation, emphasizing that STM-induced light emission is not dominated by the properties of the tip-sample cavity, but reflects the properties of the alloy particle in the tunneling contact. The deviations in the plasmon position with respect to clusters embedded in a glass matrix are attributed to the nonspherical shape of the particles and the different cluster environment in our experiment.

The major size effect we observed on single alloy clusters is an increase of the photon emission yield with increasing particle size. This behavior reflects the growing number of electrons participating in the collective oscillation. A size dependence of the plasmon position could not be detected for the analyzed alloy clusters. Two effects might be responsible for the absent shift in the plasmon energy. While a considerable blueshift of the resonance position with decreasing cluster size was observed for pure $\mathrm{Ag}$ particles on $\mathrm{Al}_{2} \mathrm{O}_{3} / \mathrm{NiAl}(110)$, a similar trend was missing in the case of $\mathrm{Au}$ particles. ${ }^{21} \mathrm{An}$ intermixing of even small amounts of $\mathrm{Au}$ into $\mathrm{Ag}$ particles could therefore initiate a quenching of the size evolution in the plasmon energy. In addition, the alloying process of $\mathrm{Ag}$ and $\mathrm{Au}$ may also depend on the cluster size, leading to different compositions of small and large particles. Such behavior could be the consequence of different diffusion and nucleation characteristics of both metals on the oxide film and could cover a potential size dependence of the plasmon energy.

The Ag-Au alloying in small particles yields also distinct changes in the linewidth of Mie-plasmon resonances. Measured line widths increase with decreasing $\mathrm{Ag}$ fraction from about $30 \mathrm{~nm}$ for pure $\mathrm{Ag}$ to $115 \mathrm{~nm}$ for pure Au clusters. Calculated peaks for alloy spheroids show also increasing widths with decreasing Ag fraction, except for Au-rich compositions [Fig. 4(b)]. The agreement with the experimental data is better, when the cluster environment is described as a combination of $70 \%$ vacuum and $30 \%$ alumina, which corresponds to the first model in the previous section. A consideration of the NiAl dielectric properties in the second model causes a sharp increase of the calculated widths and enlarges the discrepancy to experimental values. The effect is due to the large imaginary part of $\epsilon_{\mathrm{NiAl}}(\omega)$, which increases the absorption efficiency of the cluster environment and induces additional line broadening. Whereas experimental plasmon lines continuously broaden with increasing Au content in the particles, the model calculations predict decreasing line widths for particles containing more than $70 \% \mathrm{Au}$. The deviation was earlier attributed to a pronounced maximum in the imaginary part of the NiAl dielectric function at 500 $\mathrm{nm} .{ }^{34}$ At this wavelength, transitions from the NiAl $d$ to empty $s p$ bands above the Fermi level set in, which couple to the plasmon excitations and cause an additional line broadening. On the other hand, the particle density on the oxide surface increases with increasing Au content. A higher density leads to more pronounced cluster-cluster interactions in Au-rich particle ensembles, stimulating a further increase in linewidth compared to Ag-rich preparations. The chosen model allows only for a rather poor reproduction of plasmon linewidths as a function of alloy composition, most likely due to the insufficient description of the cluster environment 


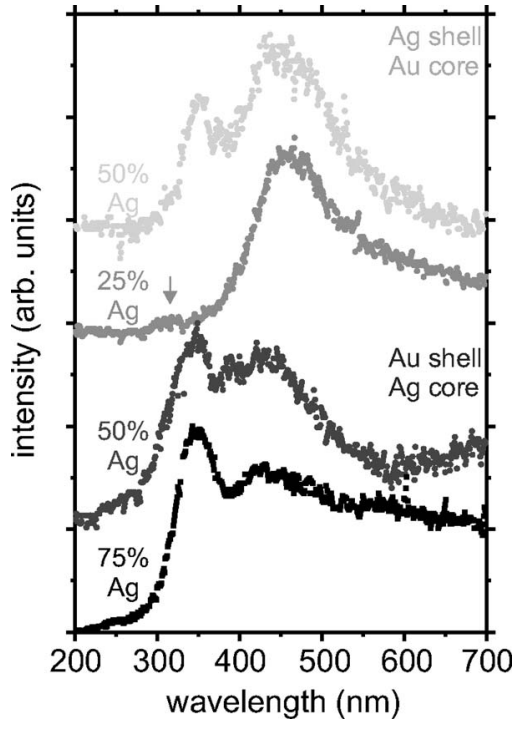

FIG. 5. Photon emission spectra of Ag shell-Au core (upper two spectra) and $\mathrm{Au}$ shell-Ag core (lower two spectra) clusters on $\mathrm{Al}_{2} \mathrm{O}_{3} / \mathrm{NiAl}(110)$. The $\mathrm{Ag}$ content of the shell-core clusters is given in the image $\left(U_{\text {tip }}<-13 \mathrm{~V}, I=4 \mathrm{nA}\right)$.

as a homogeneous medium. Furthermore, the model neglects the influence of the STM tip and cluster-cluster interactions on plasmon excitations in a single particle.

\section{Optical properties of Ag-Au shell-core clusters}

Photon emission spectra of single Ag shell-Au core and $\mathrm{Au}$ shell-Ag core clusters with two different shell to core volume ratios are shown in Fig. 5. The emission spectra clearly differ from those of the alloy particles. At equal amounts of $\mathrm{Ag}$ and $\mathrm{Au}$, the spectra exhibit two distinct maxima in the emission intensity, corresponding to shell and core excitations, respectively. For Ag shell-Au core clusters, the emission peaks are centered at $335 \pm 25 \mathrm{~nm}$ and $460 \pm 25 \mathrm{~nm}$, as determined from averaging dozens of single cluster spectra. For the reverse configuration (Au shell-Ag core), averaged peak positions at $390 \pm 35 \mathrm{~nm}$ and $435 \pm 25 \mathrm{~nm}$ were found. The peak at lower wavelength can be assigned to Ag, the one at higher wavelength to Au. Reducing the thickness of the shell leads to a decrease of the corresponding peak intensity. In the case of a very thin Ag shell, the respective emission peak shrinks to a shoulder next to the broad Au peak (see arrow in Fig. 5, second spectrum). For shell-core clusters with $25 \%$ shell volume, emission peaks were found at $325 \pm 25 \mathrm{~nm}$ and $465 \pm 20 \mathrm{~nm}$ for $\mathrm{Ag}$ shell-Au core clusters and at $370 \pm 35 \mathrm{~nm}$ and $450 \pm 25 \mathrm{~nm}$ for the reverse configuration. The separation between shell and core emission peaks increases as the shell thickness decreases for both configurations. The emission peak attributed to Ag slightly blueshifts, while the Au peak experiences a redshift. In general, the observed wavelength difference between the two emission peaks is larger for Ag shell-Au core than for Au shell-Ag core clusters. Also the widths of both emission peaks in shell-core clusters are very different. The peak associated with $\mathrm{Au}$ has a larger FWHM of approximately $100 \mathrm{~nm}$ compared to the Ag-derived peak with $50 \mathrm{~nm}$ FWHM. In addition to photon emission spectra with two distinct peaks, some of the analyzed clusters show only one maximum in their spectra. This emission peak is located at intermediate positions between the pure $\mathrm{Ag}$ and $\mathrm{Au}$ values, being very similar to the observations on alloy clusters.

An estimation of the optical properties of shell-core clusters is again obtained from calculating the polarizability of spheroids with different shell and core materials immersed in a dielectric surrounding ${ }^{10,35}$

$$
\alpha_{(1,0)}(\omega) \propto \frac{\left(\epsilon_{s}-\epsilon_{m}\right)\left(\epsilon_{s}+\left(\epsilon_{c}-\epsilon_{s}\right)\left(L_{c}^{\perp}-f L_{s}^{\perp}\right)\right)+f \epsilon_{s}\left(\epsilon_{c}-\epsilon_{s}\right)}{\left[\epsilon_{s}+\left(\epsilon_{c}-\epsilon_{s}\right)\left(L_{c}^{\perp}-f L_{s}^{\perp}\right)\right]\left[\epsilon_{m}+\left(\epsilon_{s}-\epsilon_{m}\right) L_{s}^{\perp}\right]+f L_{s}^{\perp} \epsilon_{s}\left(\epsilon_{c}-\epsilon_{s}\right)} .
$$

The same value was chosen for core $\left(L_{c}^{\perp}\right)$ and shell $\left(L_{s}^{\perp}\right)$ geometrical factors, determined in a similar way as for the alloy particles from measured aspect ratios. ${ }^{35}$ The quantity $f$ is defined as the ratio between the core and the total cluster volume: $f=\left(V_{\text {core }} / V_{\text {total }}\right) . \epsilon_{s}(\omega)$ and $\epsilon_{c}(\omega)$ depict the dielectric functions of the shell and core materials. ${ }^{36}$ As for the alloy particles, the cluster environment is described by two dielectric functions, composed of (i) $0.7 \epsilon_{\mathrm{vac}}+0.3 \epsilon_{\mathrm{Al}_{2} \mathrm{O}_{3}}$ and (ii) $0.7 \epsilon_{\mathrm{vac}}+0.15 \epsilon_{\mathrm{Al}_{2} \mathrm{O}_{3}}+0.15 \epsilon_{\mathrm{NiAl}}(\omega) .{ }^{33}$ Two maxima were obtained in the polarizability of shell-core clusters, reflecting the position of the two plasmon modes. Comparison of calculated and measured wavelength positions has to consider that the structure of the particles on the $\mathrm{Al}_{2} \mathrm{O}_{3}$ support might be better described by one material capping the other than by a closed shell-core configuration. Some general conclusions can, however, be drawn from the model calculations. For Ag shell-Au core clusters with a Ag fraction of $25 \%$, two plasmon modes have been calculated at $330 \mathrm{~nm}$ (shell) and 485 $\mathrm{nm}$ (core), leading to a mode splitting of approximately 155 $\mathrm{nm}$. Consideration of the $\mathrm{NiAl}$ dielectric properties slightly enlarges this wavelength difference. For an increasing shell thickness, the computed Ag shell mode shifts to the red, while the Au core mode experiences a blueshift, resulting in a smaller wavelength difference of $145 \mathrm{~nm}$. The experimental trend obtained from analyzing many single cluster spectra follows quite well the calculated behavior. For Ag shell-Au core clusters with $25 \% \mathrm{Ag}$, a wavelength difference of $140 \pm 35 \mathrm{~nm}$ has been determined between both modes, which decreases to $125 \pm 35 \mathrm{~nm}$ for clusters containing $50 \%$ $\mathrm{Ag}$. This indicates that the assumed Ag shell-Au core struc- 
ture is a reasonable description of the experimental situation and reflects the measured emission properties. A strong intermixing between core and shell material can be excluded for the Ag shell-Au core clusters.

The calculated optical properties of $\mathrm{Au}$ shell-Ag core clusters show a similar behavior to clusters with reverse configuration. The wavelength difference between the two plasmon modes is smallest for ultra-thin Au shells and continuously increases with increasing $\mathrm{Au}$ amount in the cluster. At a $\mathrm{Ag}$ content of $50 \%$ the wavelength difference has been determined to $150 \mathrm{~nm}$, decreasing to $140 \mathrm{~nm}$ for clusters containing $75 \%$ silver. However, the measurements reveal a considerably smaller gap between the two emission maxima, which amounts to $45 \pm 40$ and $80 \pm 40 \mathrm{~nm}$ for clusters with 50 and $75 \% \mathrm{Ag}$ content, respectively. The distinct deviation between experimental findings and computed trends in the case of $\mathrm{Au}$ shell- $\mathrm{Ag}$ core clusters indicates a strong intermixing between core and shell materials at the interface.

Gold diffusion into the Ag core would initiate a red shift of the core plasmon, while penetration of $\mathrm{Ag}$ atoms into the shell increases the respective shell plasmon energy. Both processes would reduce the gap between the plasmon modes, leading to a better agreement with the experimental values. The Ag diffusion might be the dominant mechanism due to the smaller surface free energy of $\operatorname{Ag}(111)$ and the higher diffusivity of $\mathrm{Ag}$ atoms in $\mathrm{Ag}$ and $\mathrm{Au}$ matrices. ${ }^{37,38}$ Such an interpretation is consistent with Auger-electron-spectroscopy experiments, which revealed an enhanced diffusion of $\mathrm{Ag}$ atoms through a thin $\mathrm{Au}$ film deposited on $\mathrm{Ag}(111)$ at $75^{\circ} \mathrm{C} .{ }^{39}$ Efficient diffusion at the interface of $\mathrm{Au}$ shell-Ag core colloids was earlier observed by TEM and electrondiffraction experiments. ${ }^{40}$ For Ag-coated $\mathrm{Au}$ particles in aqueous solution, the alloying rate was measured by $\mathrm{x}$-ray absorption fine structure spectroscopy and found to be related to the presence of vacancies at the interface. ${ }^{18}$ Optical measurements on ensembles of Ag-Au shell-core colloids in solution also revealed two extinction peaks, indicating independent plasmon excitations in shell and core of the particles. ${ }^{12,13,16}$ As in the present work, the Au shell-Ag core particles showed less defined optical extinction spectra compared to the opposite configuration, which again suggests enhanced diffusion of Ag atoms towards the cluster surface. Taking these arguments into consideration, an enrichment of $\mathrm{Ag}$ atoms in the Au shell provides a plausible explanation for the reduced wavelength difference between shell and core plasmon peaks observed in this experiment. The presence of clusters with only one emission peak even after successive deposition of $\mathrm{Ag}$ and $\mathrm{Au}$ provides a further indication for the efficient alloying process in shell-core clusters at room temperature. Since we analyze individual clusters in our spatially resolved experiment, we can clearly distinguish between completely alloyed particles and clusters with a remaining shell-core structure on the oxide surface.

\section{CONCLUSIONS}

We have analyzed the alloying of $\mathrm{Ag}$ and $\mathrm{Au}$ in individual alumina-supported clusters using the light emission stimulated by the tunneling current from an STM tip. For simultaneous deposition of $\mathrm{Ag}$ and $\mathrm{Au}$ atoms onto the surface, one emission peak is detected in the alloy particles, which is assigned to the $(1,0)$ Mie-plasmon resonance. With increasing $\mathrm{Ag}$ content in the clusters, the plasmon position shifts from the pure $\mathrm{Au}$ to the Ag value, following the calculated polarizability of alloy spheroids. Two distinct emission peaks are observed, when $\mathrm{Ag}$ and $\mathrm{Au}$ are successively deposited onto the $\mathrm{Al}_{2} \mathrm{O}_{3} / \mathrm{NiAl}(110)$ surface. The peaks are attributed to independent Mie-plasmon excitations in the shell and core of the clusters. The smaller wavelength splitting between the two modes in $\mathrm{Au}$ shell-Ag core clusters compared to the reverse configuration indicates a high degree of alloying in particles with Ag cores. Our experimental results on single oxide-supported Ag-Au clusters are in line with comparable ensemble measurements, demonstrating that light emission spectroscopy with the STM is a suitable technique to characterize the optical properties of individual particles.

\section{ACKNOWLEDGMENT}

Financial support of the Fonds der Chemischen Industrie is gratefully acknowledged.
*Electronic address: nilius@ @ fhi-berlin.mpg.de

${ }^{1}$ G. Ertl, H. Knözinger, and J. Weitkamp, Handbook of Heterogeneous Catalysis (Wiley, Weinheim, 1997).

${ }^{2}$ J. Sinfelt, Bimetallic Catalysts (Wiley, London, 1983).

${ }^{3}$ D. Kondarides and X. E. Verykios, J. Catal. 158, 363 (1996).

${ }^{4}$ A. Venezia, V. La Parola, G. Deganello, B. Pawelec, and J. Fierro (unpublished).

${ }^{5}$ Y.-F. Han, D. Kumar, C. Sivadinarayana, A. Clearfield, and D. Goodman, Catal. Lett. 94, 131 (2004).

${ }^{6}$ A. Molenbroek, J. Nørskov, and B. Clausen, J. Phys. Chem. B 105, 5450 (2001).

${ }^{7}$ A. Carlsson, M. Bäumer, T. Risse, and H.-J. Freund, J. Chem. Phys. 119, 10885 (2003).

${ }^{8}$ A. Carlsson, M. Naschitzki, M. Bäumer, and H.-J. Freund, J.
Phys. Chem. B 107, 778 (2003).

${ }^{9}$ A. Santra, F. Yang, and D. Goodman, Surf. Sci. 548, 324 (2004).

${ }^{10}$ U. Kreibig and M. Vollmer, Optical Properties of Metal Clusters, Vol. 25 of Springer Series Materials Science (Springer-Verlag, Berlin, 1995).

${ }^{11}$ G. Papavassiliou, J. Phys. F: Met. Phys. 6, L103 (1976).

${ }^{12}$ J. Sinzig, U. Radtke, M. Quinten, and U. Kreibig, Z. Phys. D: At., Mol. Clusters 26, 242 (1993).

${ }^{13}$ J. Sinzig and M. Quinten, Appl. Phys. A: Solids Surf. 58, 157 (1994).

${ }^{14}$ P. Mulvaney, M. Giersig, and A. Henglein, J. Phys. Chem. 97, 7061 (1993).

${ }^{15}$ S. Link, Z. Wang, and M. El-Sayed, J. Phys. Chem. B 103, 3529 (1999). 
${ }^{16}$ J. Hodak, A. Henglein, M. Giersig, and G. Hartland, J. Phys. Chem. B 104, 11708 (2000).

${ }^{17}$ M. Moskovits, I. Srnová-Šloufová , and B. Vlčková, J. Chem. Phys. 116, 10435 (2002).

${ }^{18}$ T. Shibata, B. Bunker, Z. Zhang, D. Meisel, C. Vardeman, and J. Gezelter, J. Am. Chem. Soc. 124, 11989 (2002).

${ }^{19}$ H. Shi, L. Zhang, and W. Cai, J. Appl. Phys. 87, 1572 (2000).

${ }^{20}$ M. Gaudry, J. Lermé, E. Cottancin, M. Pellarin, J.-L. Vialle, M. Broyer, B. Prével, M. Treilleux, and P. Mélinon, Phys. Rev. B 64, 085407 (2001).

${ }^{21}$ N. Nilius, N. Ernst, and H.-J. Freund, Phys. Rev. Lett. 84, 3994 (2000).

${ }^{22}$ N. Nilius, A. Cörper, G. Bozdech, N. Ernst, and H.-J. Freund, Prog. Surf. Sci. 67, 99 (2001).

${ }^{23}$ R. Jaeger, H. Kuhlenbeck, H.-J. Freund, M. Wuttig, W. Hoffmann, R. Franchy, and H. Ibach, Surf. Sci. 259, 235 (1991).

${ }^{24}$ M. Bäumer and H.-J. Freund, Prog. Surf. Sci. 61, 127 (1999).

${ }^{25}$ The amount of deposited material was calibrated by evaporating silver and gold on bare $\mathrm{NiAl}(110)$, where both metals grow in a layer by layer fashion and determination of the coverage becomes possible from STM images.

${ }^{26}$ All cluster diameters are upper limits due to tip convolution effects. The real size was estimated to be smaller by a factor of approximately 1.5 .

${ }^{27}$ K. Hojrup Hansen, T. Worren, S. Stempel, E. Lægsgaard, M. Bäumer, H.-J. Freund, F. Besenbacher, and I. Stensgaard, Phys. Rev. Lett. 83, 4120 (1999).
${ }^{28}$ F. Baletto, C. Mottet, and R. Ferrando, Surf. Sci. 446, 31 (2000).

${ }^{29}$ R. Berndt, J. K. Gimzewski, and P. Johansson, Phys. Rev. Lett. 67, 3796 (1991).

${ }^{30}$ K. Ripken, Z. Phys. 250, 228 (1972).

${ }^{31}$ K.-H. Hellwege and J. Olsen, Numerical Data and Functional Relationships in Science and Technology, Vol. 15b of LandoltBörnstein (Springer-Verlag, Berlin, 1985).

${ }^{32}$ H. Jacobi and R. Stahl, Z. Metallkd. 60, 106 (1969).

${ }^{33} \mathrm{We}$ are aware that the presence of an embedding medium with partially absorbing properties is not inline with general assumptions of the Mie theory. Introducing of $15 \% \mathrm{NiAl}$ into the averaged dielectric function can therefore only qualitatively consider the influence of a metallic support on the emission spectra.

${ }^{34}$ N. Nilius, N. Ernst, and H.-J. Freund, Surf. Sci. 478, L327 (2001).

${ }^{35} \mathrm{C}$. Bohren and D. Huffman, Absorption and Scattering of Light by Small Particles (Wiley, New York, 1983).

${ }^{36}$ E. D. Palik, Handbook of Optical Constants of Solids (Academic, Orlando, 1985).

${ }^{37}$ S. M. Foiles, M. Baskes, and M. Daw, Phys. Rev. B 33, 7983 (1986).

${ }^{38}$ H. Mehrer, Diffusion in Solid Metals and Alloys, Vol. 26 of Landolt-Börnstein (Springer-Verlag, Berlin, 1990).

${ }^{39}$ A. Bukaluk, M. Rozwadowski, and R. Siuda, Appl. Phys. A 34, 193 (1984).

${ }^{40}$ I. Srnová-Šloufová, F. Lednický, A. Gemperle, and J. Gemperlová, Langmuir 16, 9928 (2000). 